\title{
Ultra-wide bandgap semiconductor behavior of NaCaF3 fluoro-perovskite with external static isotropic pressure and its impact on optical properties: First-principles computation
}

\section{Syed Sajid Ali Gillani ( $\nabla$ dr.sajidgillani@gcu.edu.pk)}

Government College University Lahore Faculty of Science and Technology https://orcid.org/00000002-4678-4758

\section{Nisar Fatima}

University of Gujrat Faculty of Sciences

\section{Shakil}

University of Gujrat Faculty of Sciences

\section{R. Kiran}

Government College University Lahore

M. B. Tahir

University of Gujrat Faculty of Sciences

\section{A. Jawad}

Government College University Lahore

Riaz Ahmad

Government College University Lahore

\section{Research Article}

Keywords: Fluoro-perovskite, First-principles computation, Ultra-wide bandgap, Structural optimization, Electronic band transition, Refractive index, Blue-shift in loss function

Posted Date: April 14th, 2021

DOI: https://doi.org/10.21203/rs.3.rs-336152/v1

License: (c) (i) This work is licensed under a Creative Commons Attribution 4.0 International License. Read Full License 


\title{
Ultra-wide bandgap semiconductor behavior of $\mathrm{NaCaF}_{3}$ fluoro- perovskite with external static isotropic pressure and its impact on optical properties: First-principles computation
}

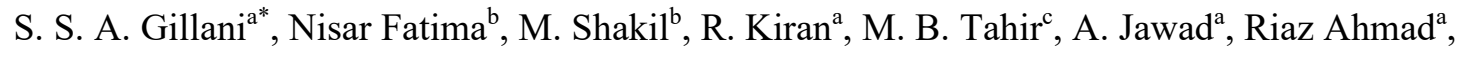 \\ ${ }^{a}$ Department of Physics, Government College University Lahore, Lahore 54000, Pakistan \\ ${ }^{b}$ Department of Physics, University of Gujrat, Gujrat 50700, Pakistan \\ ${ }^{c}$ Department of Physics, Khawaja Fareed University of Engineering and Information Technology Rahim \\ Yar Khan, Rahim Yar Khan 64200, Pakistan \\ *Corresponding author: S. S. A. Gillani (dr.sajidgillani@gcu.edu.pk)
}

\begin{abstract}
A comprehensive theoretical study to investigate the outcomes of externally applied static isotropic pressure $(0 \mathrm{GPa}-50 \mathrm{GPa})$ on electronic, optical and structural properties of $\mathrm{NaCaF}_{3}$, using density functional theory (DFT) based CASTEP (Cambridge Serial Total Energy Package) code with ultra-soft pseudo-potential USP plane wave and Perdew Burke Ernzerhof (PBE) exchange-correlation functional of Generalized Gradient Approximation (GGA), is reported. The electronic bandgap shows the increasing trend $4.773 \mathrm{eV}-6.203$ $\mathrm{eV}$ (direct bandgap) with increasing external pressure. The increase in bandgap is significant up to $20 \mathrm{GPa}$ as compared to higher external pressures. The mystery of increasing band gap is nicely decoded by total density of states (TDOS) and elemental partial density of states (EPDOS). Optical properties have been calculated to analyze the impact of increment in band gap on them. We observed that highest peak of energy loss function $L(\omega)$ shows the blue shift which confirms the increment of band gap. At zero photon energy, for $0 \mathrm{GPa}$, the static refractive index $n(\omega)$ has value of 1.4456. After applying external pressure, there is a slight increase in $n(\omega)$ which favors the semiconducting behavior of ternary compound. The energy points at which the absorption peak is maxima, the refractive index has lowest value.
\end{abstract}

Keywords: Fluoro-perovskite; First-principles computation; Ultra-wide bandgap; Structural optimization; Electronic band transition; Refractive index, Blue-shift in loss function. 


\section{Introduction}

After the very first discovery of perovskites in 1839 , many materials have been discovered carrying the same crystalline structure, from the list one of the most common material is $\mathrm{CaTiO}_{3}$. The structural composition of cations and anion in some particular order leads to the basic structure of any perovskites defined by the general formula of $\mathrm{ABX}_{3}$. Here $\mathrm{A}$ and $\mathrm{B}$ are cations, and mostly they belong to alkali metals or alkaline earth metals, whereas $\mathrm{X}$ is the anion commonly oxygen has been used in place of $\mathrm{X}$ [1]. However, the halogen perovskites have also drawn attention $\mathrm{X}$ is replaced by an element of group VII-A (most commonly Fluorine $\mathrm{F}$ ). This $\mathrm{X}$ is further attached with mono-valent $\mathrm{A}$ and divalent $\mathrm{B}$ cations in a quite attractive yet technical way. This stoichiometry then exposes numerous electronic, magnetic, and optical changes in response to a variety of temperature and pressure [2].

Fluoride perovskites exhibits a quite wide bandgap which helps then to become suitable for being applied in lenses, ferroelectric, antiferromagnetic systems and optical technologies such as transparent and optical coatings. These applications make $\mathrm{KCaF}_{3}$, $\mathrm{RbCaF}_{3}, \mathrm{BaLiF}_{3}$ and $\mathrm{SrLiF}_{3}$ and some other members of this peculiar class of enormous interest [3] but still more and more investigations are required to be done both experimentally and theoretically. Their magnetic, electronic, and optical properties are catching all the attention as they show promising features when it comes to these properties. Research has explored perovskites showing superconductivity, piezoelectric effect, ferroelectricity, magneto resistance and some highly beneficial thermal properties and yet quite more to find out to make these properties useful in a broad way [4-7]. Their wide bandgap is also their key feature allowing them to be used in lenses, memory devices, sensors, fuel cells whereas the absorption property leads to LEDs and colors TVs.

Changing different variables explore different properties and variation in any material. As long as perovskites are concerned, research has shown by varying some parameters lead to a whole set of varied properties. For instance, by applying pressure or thermal conditions we may know how the material responds. The purpose of varying these parameters is to know the elastic limit, sheer (shear) strength, bulk modulus, conductivity, magnetic 
behavior, energy band gap and a lot of other properties. For $\mathrm{KCaX}_{3}(\mathrm{X}=\mathrm{F}, \mathrm{Cl})$, theoretical study was carried to unveil elastic, optical, and electronic behavior in linear density functional theory. The indirect bandgap for $\mathrm{KCaX}_{3}(\Gamma-\mathrm{R})$ was revealed. Moreover, the linear relation of Electronic bandgap and hydrostatic pressure was also a result of the study [8]. For $\mathrm{KCaF}_{3}$ optimization method provided derivation for Bulk modulus, pressure and equilibrium lattice parameters. Elastic moduli were seemed to be pressure dependent along with the directional dependence of Young's Moduli, whereas the pressure was provided in the range of 0-40 GPa. A linear dependence of Energy band gap on pressure was also shown, however, different techniques gave different results for the value of band gap, but the main value is $6.1 \mathrm{eV}$ in $\mathrm{R}-\Gamma$, which varied on providing pressure [9].

Another perovskite, $\mathrm{BaZrO}_{3}$ is also famous for its diverse properties and have been investigated with and without doping and under high pressure [10]. An experimental study showed that the cubic structure of $\mathrm{BaZrO}_{3}$ was transformed to tetragonal phase on applying high pressure. The provided pressure was $17.2 \mathrm{GPa}$ on which the phase transition occurred [11]. Later a theoretical research was carried to investigate the accuracy experimental results. The material was studied in density functional theory, and it was found that material carries the indirect band-gap for both tetragonal and cubic structures. And after the phase transition from Cubic to tetragonal there was merely $1 \mathrm{eV}$ increment in the bandgap. So, the results were in the favor of the previous experimental literature. The mechanical stability of both tetragonal and cubic phases was also inferred [12]. Another research explored the electronic, elastic, optical and structural properties of $\mathrm{LiBeF}_{3}$, showing the indirect bandgap of this perovskites. The reported value of bandgap was $7.83 \mathrm{eV}$. Whereas the absorption value of this material lies in ultraviolet region [13].

Under discussion material is $\mathrm{NaCaF}_{3}$ (NCF) which is also a fluro- perovskite following the same structure, here sodium is alkali metal carrying positive charge and is mono-valent cation. On the other hand, $\mathrm{Ca}$ is divalent cation, and is alkaline earth metal possessing positive charge. For the purpose of stability B positioned ions are greater than the ions at A position. Whereas the highly electronegative element fluorine belongs to the family of halogens, i. e. Group VII-A, and the value for its electronegativity is 4 [14]. Fluorides based 
on alkali metals and alkaline earth metals have attained limelight because of their ferromagnetic, piezoelectric and photoluminescence properties [15].

Currently the optical, electronic, and structural properties of $\mathrm{NaCaF}_{3}$ have been explored by applying different external isotropic static pressure. Moreover, by the end of the paper some questions will be answered, how changing the pressure is affecting the bandgap of this perovskite and whether or not by applying pressure the materials conducting behavior changes from semiconductor to insulator or it shows ultra-wide bandgap semiconductor nature?

\section{Computational methodology}

For ternary compound $\mathrm{NaCaF}_{3}$, having cubic structure of $P m 3 m$ space group has been considered. The settlement for $\mathrm{Ca}$ and $\mathrm{Na}$ atoms is $(0,0,0)$ and $(1 / 2,1 / 2,1 / 2)$ respectively, however the three $\mathrm{F}$ atoms are at $(1 / 2,1 / 2,0),(1 / 2,0,1 / 2),(0,1 / 2,1 / 2)$. The configuration

of valence electrons for $\mathrm{NaCaF}_{3}$ compound is given as: $\mathrm{F}$ has $2 s^{2}, 2 p^{5}$, while $\mathrm{Na}$ has $2 s^{2}$, $2 p^{6}, 3 s^{1}$ and $\mathrm{Ca}$ has $3 s^{2}, 3 p^{6}, 4 s^{2}$.

In this paper, the technique of ultra-soft pseudo-potential USP plane wave $[16,17]$ and Perdew Burke Ernzerhof (PBE) [18, 19] exchange correlation functional of GeneralizedGradient-Approximation (GGA) has been applied, based on density functional theory (DFT), for the calculations of structural, electronic and optical properties, employed in CASTEP (Cambridge Serial Total Energy Package) code [20]. All these calculations have been done by solving Kohn-Sham equations [21-23]. By implementing this method; both quick and efficient computations can be achieved simultaneously, without taking into consideration the approximation of shape of orbitals in advance. The outcome of interaction of nuclei with inner shell electrons is the formation of ionic core. Then there is an interaction between valence electrons and ionic core resulting in convergence of electron-ion potential. By using special $k$-points, integrations have been performed in the Brillion zone, with $k$-points mesh designed in order of $8 \times 8 \times 8$ on Monkhorst pack grid for structural optimization [24]. Here the value of total energy is taken as $5 \times 10^{-5} \mathrm{eV} /$ atom. While during optimization of structure, the cell parameters and positions of atoms are varied to minimize the total energy under the prescribed boundaries of the symmetry. 
Further, all of the forces applied on the atoms are converged during geometrical optimization, to a value of $0.002 \mathrm{eV} / \AA$ [25]. All the properties of material are calculated after geometry optimization.

\section{Result and discussion}

\subsection{Structural properties}

After the geometrical optimization, the unit cell of $\mathrm{NaCaF}_{3}$ is shown in Fig.1. It appears from the figure that NCF has a cubic phase with space group $P m 3 m$. The lattice constant of NCF in cubic phase at zero pressure is $a=b=c=4.154 \AA$. As the pressure is applied in intervals $(5,10,15,20,25,30,35,40,45,50) \mathrm{GPa}$ the lattice constant appears to be (4.049, $3.964,3.903,3.848,3.805,3.765,3.731,3.696,3.668,3.643) \AA$, respectively.

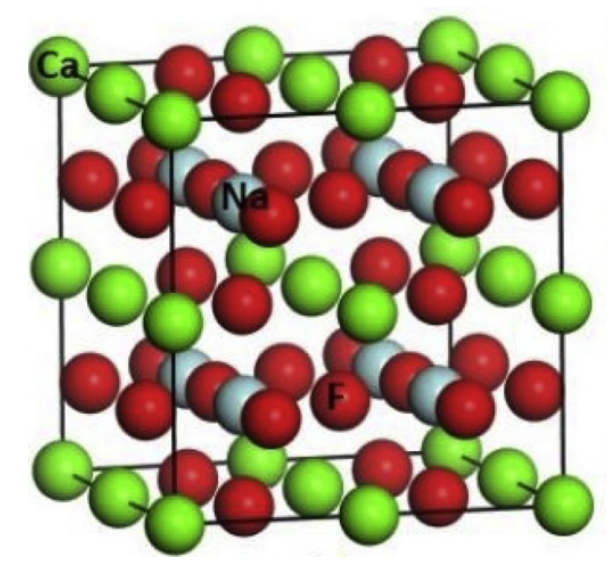

Fig. 1. $2 \times 2 \times 2$ supercell of NCF.

The decrement in lattice constant can be seen with an increment in pressure. This reduction in lattice constant by increasing pressure proposes that there exist stronger atomic interactions. Hence, the unit cell volume also reduces which is because of the decrease in lattice parameters. It has also been noticed that no literature either experimental or theoretical, for pressure induced NCF compound for evaluation. Thus, all the results calculated in this paper will be confirmed by future measurements. 
Table 1: Literature comparison of impact of pressure after geometry optimization of $\mathrm{NaCaF}_{3}$ providing volume for unit cell and parameters of lattice.

\begin{tabular}{|c|c|c|c|c|c|}
\hline NaCaF 3 & \multicolumn{3}{|c|}{ Lattice Constants } & Volume & References \\
\hline $\begin{array}{c}\text { Pressure } \\
(\text { GPa })\end{array}$ & $\boldsymbol{a}$ & $\boldsymbol{b}$ & $\boldsymbol{c}$ & $\left(\AA^{3}\right)$ & \\
\hline \multirow{3}{*}{$\mathbf{P}=\mathbf{0}$} & 4.460 & 4.460 & 4.460 & 88.717 & {$[26]$} \\
\cline { 2 - 6 } & 4.438 & 4.438 & 4.438 & 87.410 & {$[27]$} \\
\cline { 2 - 6 } & 4.378 & 4.378 & 4.378 & 83.913 & {$[27]$} \\
\cline { 2 - 6 } & 4.316 & 4.316 & 4.316 & 80.398 & {$[28]$} \\
\cline { 2 - 7 } & 4.350 & 4.350 & 4.350 & 82.313 & {$[29]$} \\
\cline { 2 - 7 } & 4.154 & 4.154 & 4.154 & 71.680 & Present work \\
\hline $\mathbf{P}=\mathbf{5}$ & 4.049 & 4.049 & 4.049 & 66.381 & Present work \\
\hline $\mathbf{P}=\mathbf{1 0}$ & 3.964 & 3.964 & 3.964 & 62.288 & Present work \\
\hline $\mathbf{P}=\mathbf{1 5}$ & 3.903 & 3.903 & 3.903 & 59.456 & Present work \\
\hline $\mathbf{P}=\mathbf{2 0}$ & 3.848 & 3.848 & 3.848 & 56.778 & Present work \\
\hline $\mathbf{P}=\mathbf{2 5}$ & 3.805 & 3.805 & 3.805 & 55.089 & Present work \\
\hline $\mathbf{P}=\mathbf{3 0}$ & 3.765 & 3.765 & 3.765 & 53.370 & Present work \\
\hline $\mathbf{P}=\mathbf{3 5}$ & 3.731 & 3.731 & 3.731 & 51.937 & Present work \\
\hline $\mathbf{P}=\mathbf{4 0}$ & 3.696 & 3.696 & 3.696 & 50.489 & Present work \\
\hline $\mathbf{P}=\mathbf{4 5}$ & 3.668 & 3.668 & 3.668 & 49.350 & Present work \\
\hline $\mathbf{P}=\mathbf{5 0}$ & 3.643 & 3.643 & 3.643 & 48.348 & Present work \\
\hline
\end{tabular}

\subsection{Electronic band structure and density of states}

The information about the levels of energy which are occupied by the electrons (Energy band) and those which are not available for the electrons to occupy i. e. zero availability (band gap) is given by the structure of electronic bands. The energy bands are of two types. One goes up beyond the Fermi level $\left(E_{\mathrm{F}}\right)$ named as conduction band $(\mathrm{CB})$ and the other one lays down the $E_{\mathrm{F}}$ named as Valence band (VB). Because all the computations have been worked out at $0 \mathrm{~K}$, therefore, without considering the finite temperature 

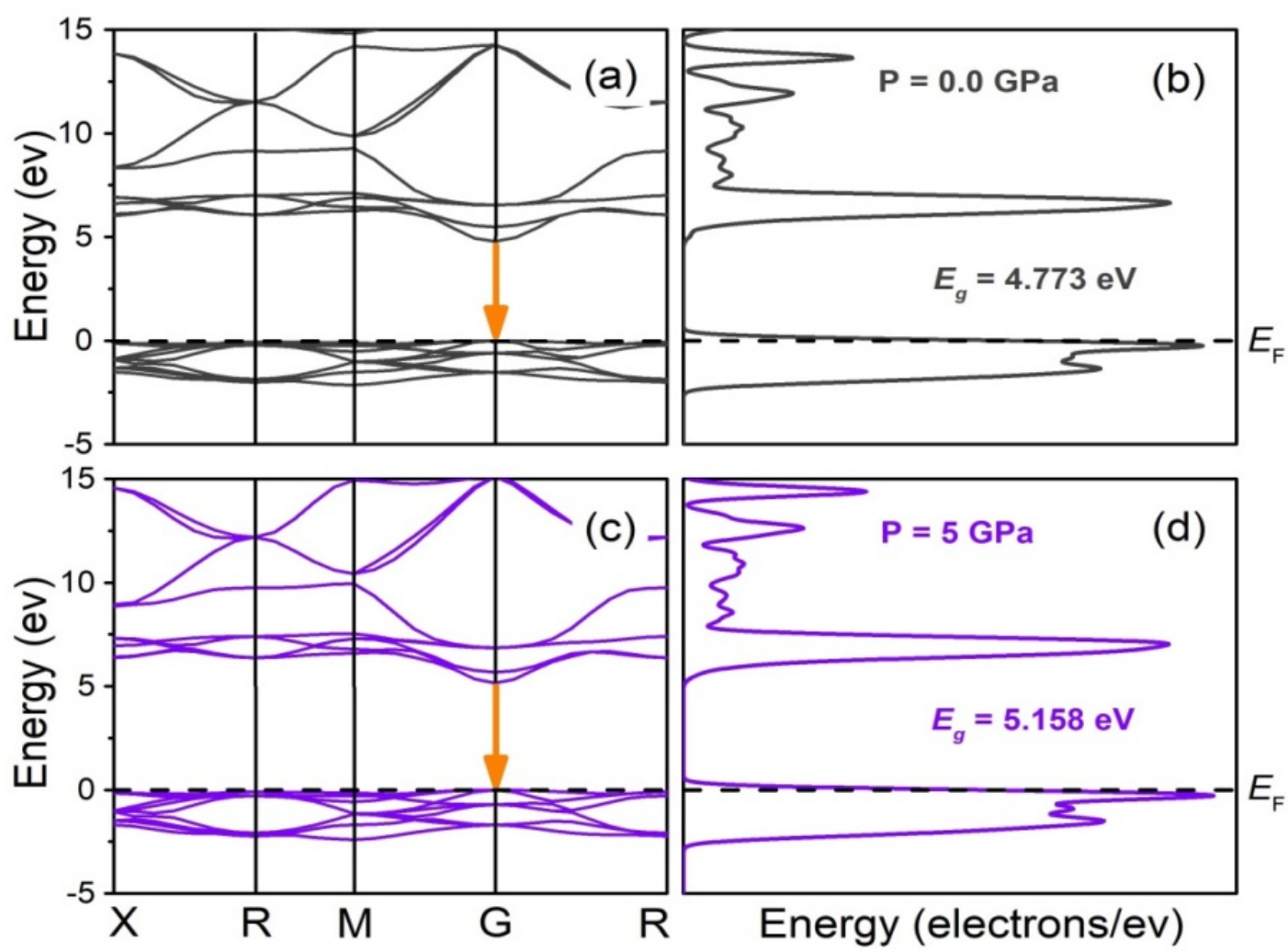

Fig. 2 At $0 \mathrm{GPa}$, (a) energy band structure and (b) DOS of NCF. At $5 \mathrm{GPa}$, (c) energy band structure and (d) DOS of NCF.

impacts, the highest point of valence band is accredited as Fermi level. The determination of band gap value by ascertaining the difference between maxima of VB and minima of $\mathrm{CB}$. If the point of symmetry of maxima of $\mathrm{VB}$ and that of minima of $\mathrm{CB}$ are upon the same point, then character of band gap would be recognized as direct band gap. However, in an inverse situation when they are not found on same point of symmetry, the gap between them can be categorized as indirect band gap.

The electronic band structure of NCF in highly symmetric Brillion zone direction is being discussed in succeeding paragraphs and shown in Fig. 2, with the Brillouin zone symmetry points displayed along the $\mathrm{x}$-axis and the energy rage $-5 \mathrm{eV}$ to $15 \mathrm{eV}$ at $\mathrm{y}$-axis was selected, under the influence of pressure ranging from $0-5 \mathrm{GPa}$. 

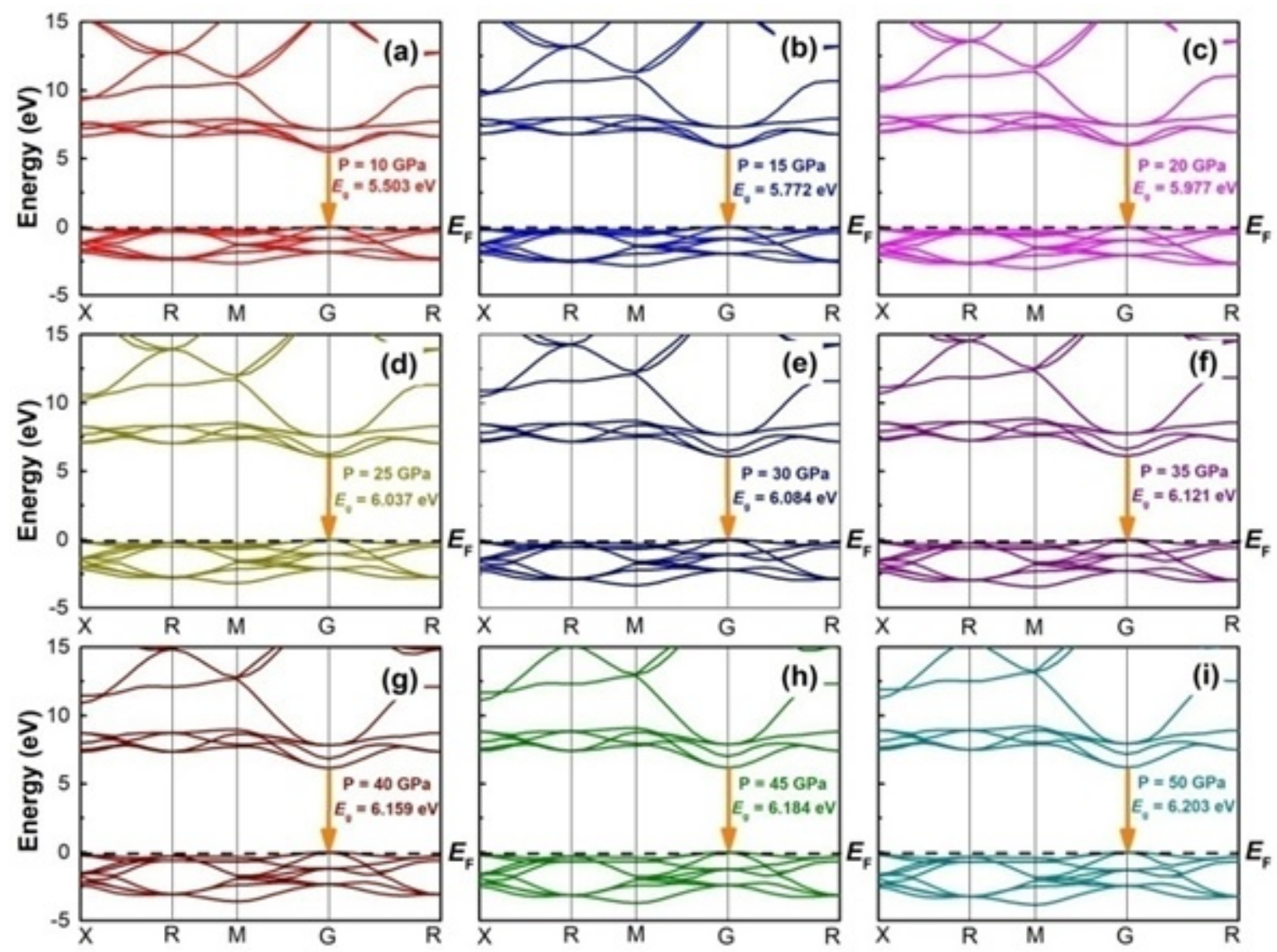

Fig. 3 (a-i) The energy band spectrum at different pressure (10 GPa - $50 \mathrm{GPa}$ ), respectively.

If $\mathrm{NCF}$ is without any pressure i. e. $\mathrm{P}=0 \mathrm{GPa}$, both the crest (maxima of $\mathrm{VB}$ ) and trough (minima of $\mathrm{CB}$ ) then find their place in Brillion zone on G-symmetry and ternary compound evinces direct band gap as shown in Fig. 2a. Arithmetically, the value of direct band gap in this case works out to $4.773 \mathrm{eV}$ behaving as a semiconductor with wide bandgap. Figure $2 \mathrm{~b}$ exhibits the graphical scheme of its density of states (DOS) illustrating that none of the states exists in this energy region.

The band gap structure and DOS for NCF at pressure $(\mathrm{P}=5 \mathrm{GPa})$ is shown in Fig. 2(c, d), respectively. In NCF at a pressure of $5 \mathrm{GPa}$, there is an increase in band gap value which appears to be $5.158 \mathrm{eV}$, with both valence band maximum (VBM) and conduction band minimum (CBM) lying on same symmetry point of Brillion zone i. e. G-symmetry point as that at zero pressure, manifesting again direct $(\mathrm{G}-\mathrm{G})$ band gap. This rise in band gap is because of the shifting of conduction band away from the Fermi level which is because of decrease in lattice constant with isotropic. 
The electronic band gap structure of NCF at pressure $(\mathrm{P}=10,15,20 \mathrm{GPa})$ is shown in Fig. $3(\mathrm{a}, \mathrm{b}, \mathrm{c})$. For NCF at pressure $10 \mathrm{GPa}, 15 \mathrm{GPa}$ and $20 \mathrm{GPa}$, there is still an increase in band gap value which is found to be $5.503 \mathrm{eV}, 5.772 \mathrm{eV}$ and $5.977 \mathrm{eV}$, respectively. Even at these pressures $(\mathrm{P}=10,15,20 \mathrm{GPa})$, band gaps appear at G-point of symmetry of Brillion zone. However, the band gap nature again appears to be direct band gap.

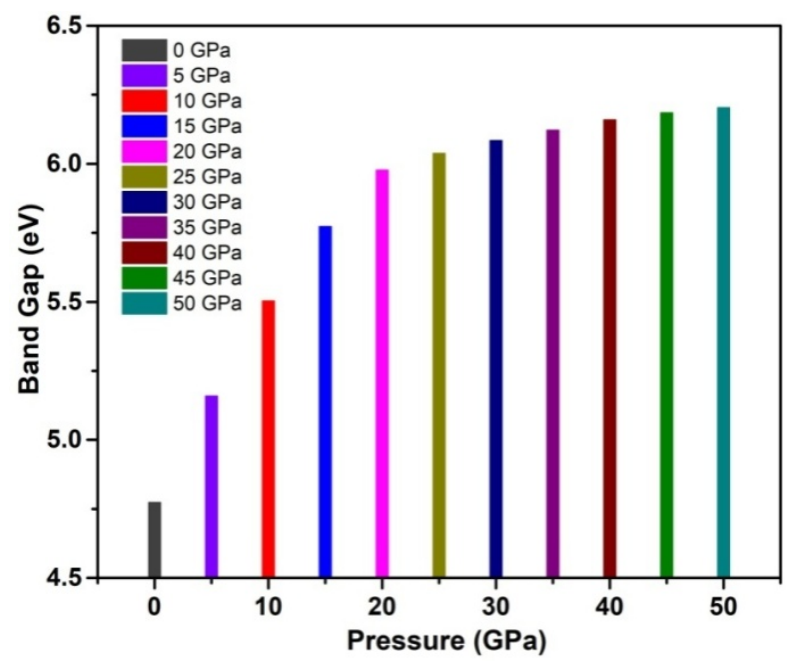

Fig. 4. Value of bandgaps of $\mathrm{NaCaF}_{3}$ with pressure which is showing increasing trend.

The band gap structure and DOS for NCF at pressure ( $\mathrm{P}=25,30,35 \mathrm{GPa})$ is shown in Fig. $3(\mathrm{~d}, \mathrm{e}, \mathrm{f})$. For NCF at pressure $25 \mathrm{GPa}, 30 \mathrm{GPa}$ and $35 \mathrm{GPa}$, there is a slight increase in band gap value which is found to be $6.037 \mathrm{eV}, 6.084 \mathrm{eV}$ and $6.121 \mathrm{eV}$, respectively. Moreover, the crest of VBM and trough of CBM do lie on G-point of symmetry under these three conditions and band gap is appeared as direct band gap.

The band gap structure and DOS for NCF at pressure $(\mathrm{P}=40,45,50 \mathrm{GPa})$ is shown in Fig. $3(\mathrm{~g}, \mathrm{~h}, \mathrm{i})$. For NCF at pressure $40 \mathrm{GPa}, 45 \mathrm{GPa}$ and $50 \mathrm{GPa}$, there is also a systematic increase in value of band gap which is computed as $6.159 \mathrm{eV}, 6.184 \mathrm{eV}$ and $6.203 \mathrm{eV}$, respectively. The material behaves as an ultra-wide bandgap semiconductor. Furthermore, the band gap appears to be a direct band gap. 

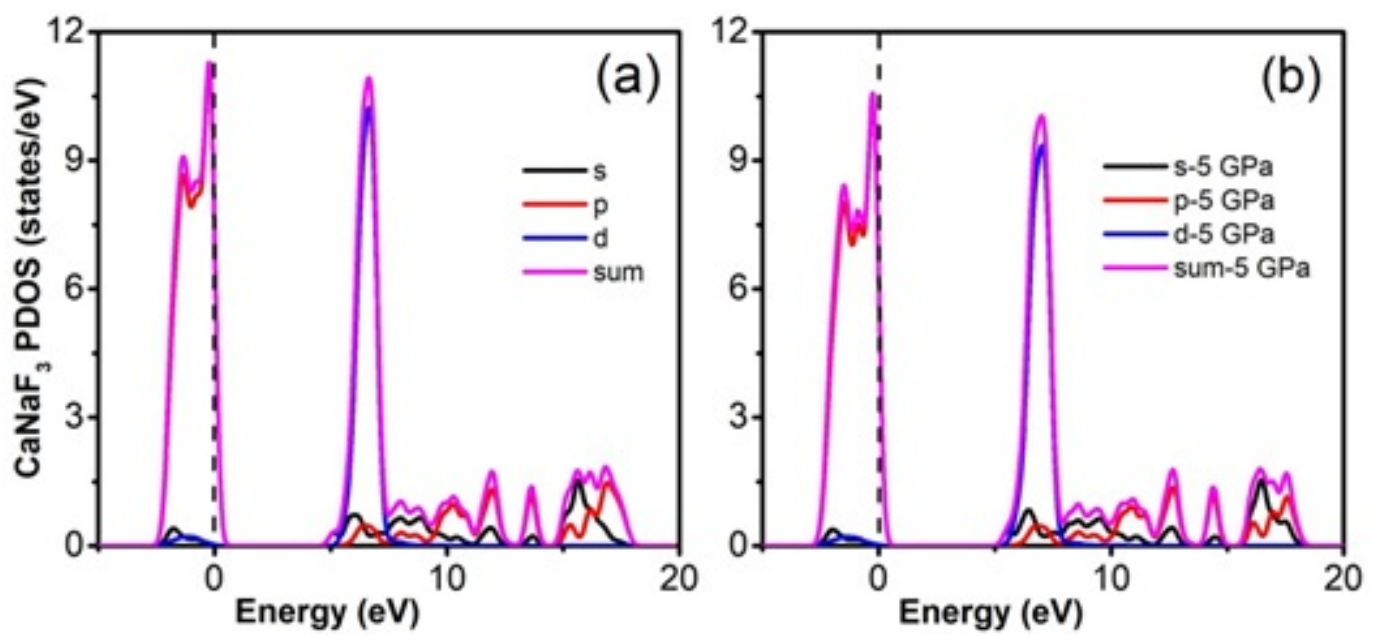

Fig. 5. Partial density of states (PDOS) of $\mathrm{NaCaF}_{3}$ at pressure (a) $0 \mathrm{GPa}$ and (b) $5 \mathrm{GPa}$.

It can be concluded from the band structure analysis that band gap is increased $(4.773 \mathrm{eV}-$ $6.203 \mathrm{eV}$ ) with static external isotropic pressure from 0GPa to 50GPa and VBM and CBM, in all cases, remains at the same symmetry point $\mathrm{G}$ which exhibits the same nature of band gap which is direct. So, we can say that pressure and band gap have direct relation (Fig. 4). The lattice parameters are also changed (Table 1) when the external pressure is applied. This change in lattice constants causes the variation of band gap at symmetry points. The external applied pressure affects the overlap integral, which causes the variation in width of band gap, as a result the electronic band structure is altered. This increase in the band gap can be explained well with three density of states DOS i. e. partial density of states (PDOS), total density of states (TDOS) and elemental partial density of sates (EDOS).

Figure $5(\mathrm{a}, \mathrm{b})$ depicts the partial density of states (PDOS) at pressure 0GPa and 5GPa and Fig. 6 (c-k) portrayed the PDOS under pressure 10 - $50 \mathrm{GPa}$ of $\mathrm{NaCaF}_{3}$. The Fermi energy $E_{\mathrm{F}}$ is shown by the vertical line in each spectrum. It can be clearly seen that in all cases $d$ states are sharper than $s$ - and $p$-states. It can be scrutinized that bellow $E_{\mathrm{F}} p$-states contribute majorly than $s$ - and $d$-states while $d$-states play important contribution above $E_{\mathrm{F}}$ than $s$ - and $p$-states. The $s$ - and $p$-states are hybridized above $E_{\mathrm{F}}$. As the external applied pressure is enhanced the hybridization between $s$ - and $p$-states is reduced so the band gap is increased progressively. 

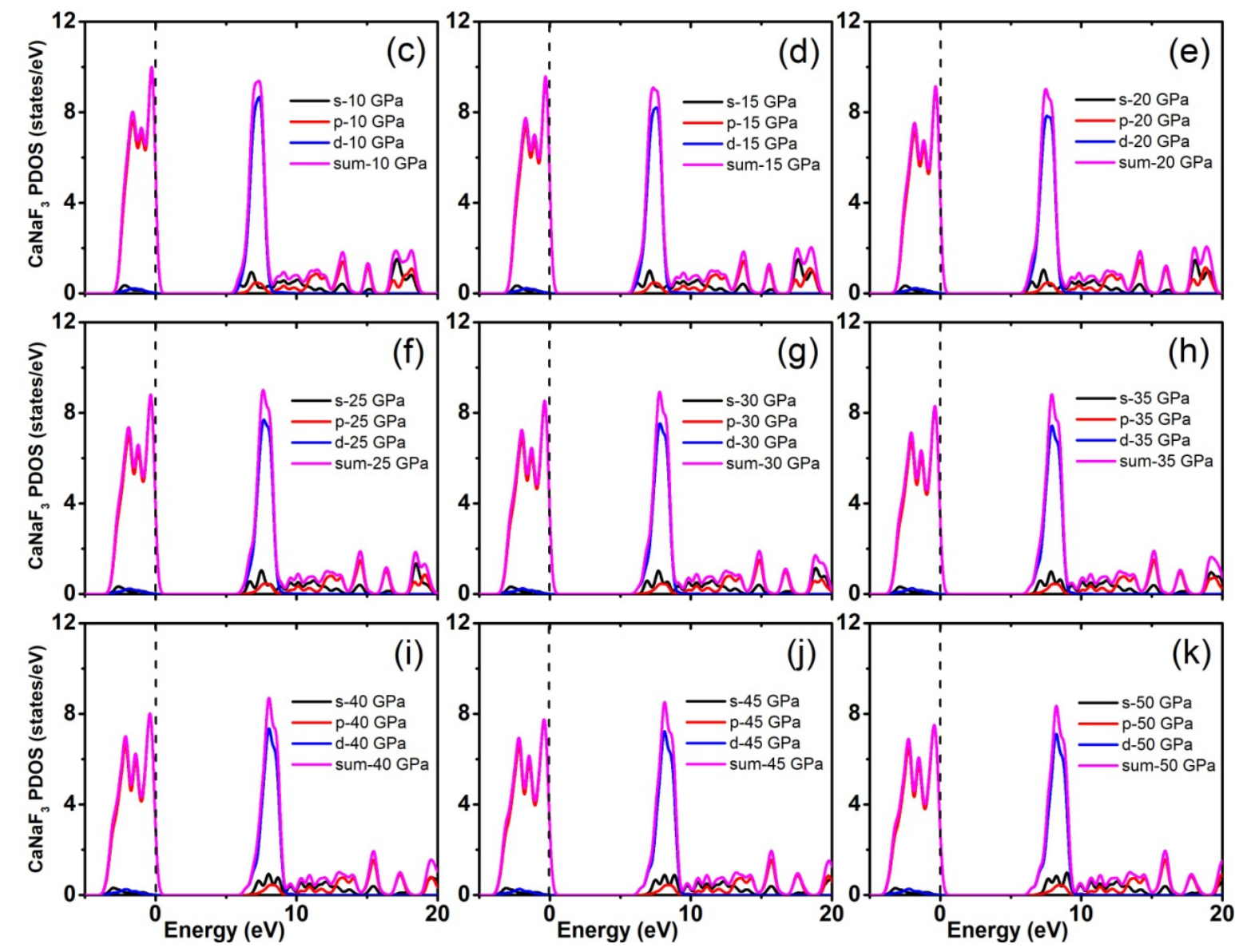

Fig. 6. Partial density of states of $\mathrm{NaCaF}_{3}$ under different pressure ranging $10 \mathrm{GPa}-50 \mathrm{GPa}$.

It can be cleared from the all spectrums that states in the valance band shifts towards lower energy such as the states in conduction band shifts towards higher energies on the application of external pressure which is the basic reason for the increment of bandgap.

It has been observed that that $\mathrm{Ca}-s$-states, F- $p$-states, Na- $s$-states, and $\mathrm{Na}$ - $p$-states exists to contribute in the upper valance band. Whereas $p$-states of $\mathrm{F}$ and $\mathrm{Na}$ perform the major role to form valence band maxima. F- $p$-states are more prominent near $E_{\mathrm{F}}$ and Na- $p$-states are prominent quite bellow $E_{\mathrm{F}}$. Furthermore, in the conduction band Ca- $p$-states, Na-s-states and Na- $p$-states are present to contribute. Here Ca- $p$-states have huge involvement in the minima of the conduction band around 5-10 eV and Na-s-states and Na-p-states are present above of conduction band around 10-20 eV. 
We can conclude from the above results that elemental PDOS is shifted to the higher and lower energies due to various pressures leads to the increment of band gap and this influenced the optical properties which are discussed below.

\subsection{Optical properties under static isotropic pressure}

The electronic structure of solids can be explained by the optical properties of a perovskite material such as function of energy loss, coefficient of absorption, reflectivity, and index of refraction as well as relative permittivity. They propose the suitability and feasibility of materials in nano-electronics and optoelectronics industries thus playing a noteworthy role [30].

All of these optical properties come into existence as a consequence of wave (Electromagnetic waves) matter (material) interaction and they are inter-related to each other, hence, complex dielectric function is used for this purpose, which is:

$$
\varepsilon(\omega)=\varepsilon_{1}(\omega)+i \varepsilon_{2}(\omega)
$$

here $\varepsilon_{1}(\omega)$ and $\varepsilon_{2}(\omega)$ symbolize the real as well as imaginary part of dielectric function, respectively. The real part of dielectric function $\varepsilon_{1}(\omega)$ provides information about intensity of polarization of a material. The value of static-dielectric function $\varepsilon_{1}(0)$ for NCF appears at $2.090 \mathrm{eV}$ at zero pressure and slightly increases towards higher value as pressure increases as shown in Fig.8a. The main peak of the real part appears after this static value and shifts towards higher energies $2.084 \mathrm{eV}, 2.108 \mathrm{eV}, 2.128 \mathrm{eV}, 2.148 \mathrm{eV}, 2.119$ $\mathrm{eV}, 2.135 \mathrm{eV}, 2.149 \mathrm{eV}, 2.166 \mathrm{eV}, 2.180 \mathrm{eV}$ and $2.194 \mathrm{eV}$ as the pressure increases from $(5,10,15,20,25,30,35,40,45,50) \mathrm{GPa}$, respectively. The complex part of dielectric function $\varepsilon_{2}(\omega)$ plays a significant character in optical properties for every material, which is related to the energy dissipation. The imaginary part of dielectric function has been exhibited in Fig. $8 \mathrm{~b}$ and its value appears to be zero at $0 \mathrm{eV}$ for NCF both in case of without and with application of high pressure. The main peak of $\varepsilon_{2}(\omega)$ for NCF at zero pressure seems about $26.840 \mathrm{eV}$ and as the rise in pressure intensity of this peak decreases with 
minor shift towards higher energy value. This main peak corresponds directly with the main peak of absorption (Fig. 8c).
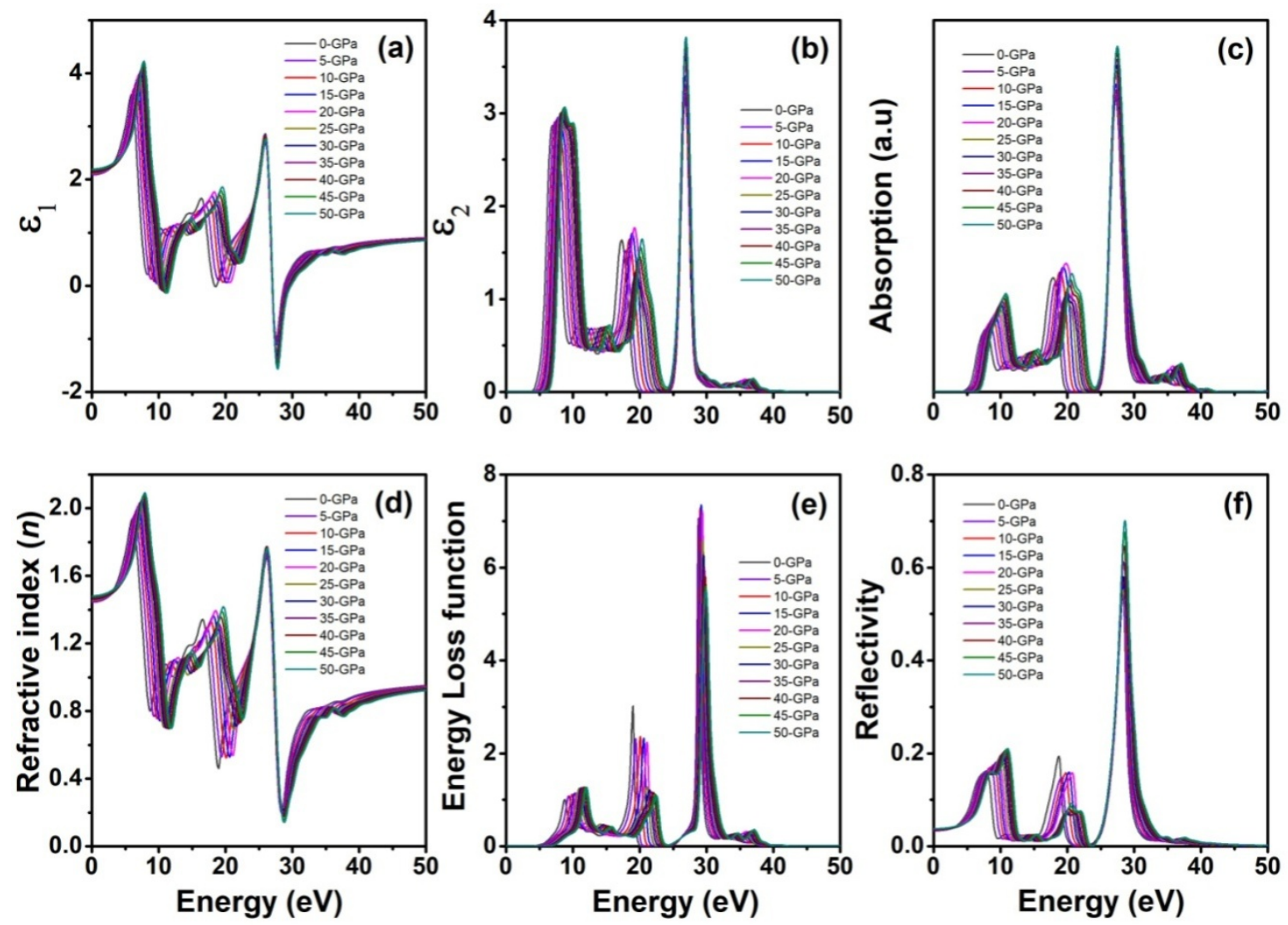

Fig. 8. Optical properties of $\mathrm{NaCaF}_{3}$ with inclusion and exclusion of a variety of pressure. (a, b) Dielectric function consist of real and imaginary part (c) absorption spectra, (d) refractive index, (e) energy-loss function and (f) reflectivity.

With pressure increase from 0 to $50 \mathrm{GPa}$, the main peaks have moved to a higher energy region from $28.2819 \mathrm{eV}, 28.3879 \mathrm{eV}, 28.5163 \mathrm{eV}, 28.5870 \mathrm{eV}, 28.5056 \mathrm{eV}, 28.3900 \mathrm{eV}$, $28.4018 \mathrm{eV}, 28.4511 \mathrm{eV}, 28.5158 \mathrm{eV}, 28.5901 \mathrm{eV}$ and $28.6534 \mathrm{eV}$, respectively.

As the pressure increases up to $20 \mathrm{GPa}$, all peaks shift to the higher energy gradually and the reflectivity increases correspondingly. Thus, blue shift takes place in whole energy region due to pressure.

To compute additional optical properties such as refractive index $n(\omega)$, energy-loss $L(\omega)$, absorption-coefficient $I(\omega)$ and reflectivity $R(\omega)$, equations given below are applied [52]; 
and the associated results have been acquired for $\mathrm{NaCaF}_{3}$ ternary compound without and with pressure ranging from (0 - 50) GPa and have been presented in Fig 8.

$$
\begin{aligned}
& n(\omega)=\left[\frac{\varepsilon_{1}(\omega)}{2}+\frac{\sqrt{\varepsilon_{1}^{2}(\omega)+\varepsilon_{1}^{2}(\omega)}}{2}\right]^{1 / 2} \\
& L(\omega)=-\operatorname{Im}\left(\varepsilon(\omega)^{-1}\right)=\frac{\varepsilon_{2}(\omega)}{\varepsilon_{1}(\omega)^{2}+\varepsilon_{2}(\omega)^{2}} \\
& I(\omega)=\sqrt{2} \omega\left[\sqrt{\varepsilon_{1}^{2}(\omega)+\varepsilon_{2}^{2}(\omega)}-\varepsilon_{1}(\omega)\right]^{1 / 2} \\
& R(\omega)=\frac{n+i k-1}{n+i k+1}
\end{aligned}
$$

Table 2: Static value at $0 \mathrm{eV}$ of $\varepsilon_{1}(\omega)$ and $n(\omega)$ and major peaks of $\varepsilon_{2}(\omega), I(\omega), L(\omega)$ and $R(\omega)$ are presented.

\begin{tabular}{|c|c|c|c|c|c|c|}
\hline Pressure & $\begin{array}{c}\text { Static } \\
\text { value } \\
\text { of } \\
\boldsymbol{\varepsilon}_{\mathbf{1}}(\boldsymbol{\omega})\end{array}$ & $\begin{array}{c}\text { Main } \\
\text { peak, } \\
\boldsymbol{\varepsilon}_{\mathbf{2}}(\boldsymbol{\omega}) \\
(\mathbf{e V})\end{array}$ & $\begin{array}{c}\text { Absorption } \\
\text { peak, } \boldsymbol{I}(\boldsymbol{\omega})\end{array}$ & $\begin{array}{c}\text { Static } \\
\text { refractive } \\
\text { index } n(\omega)\end{array}$ & $\begin{array}{c}\text { Plasmon } \\
\text { peak, } \\
\boldsymbol{L}(\boldsymbol{\omega}) \\
(\mathbf{e V})\end{array}$ & $\begin{array}{c}\text { Reflectivity } \\
\text { peak, } \boldsymbol{R}(\boldsymbol{\omega})\end{array}$ \\
\hline $\mathbf{0}$ & 2.0898 & 26.8288 & 27.2922 & 1.4456 & 28.7962 & 28.2819 \\
\hline $\mathbf{5}$ & 2.0999 & 26.8128 & 27.3243 & 1.4467 & 28.9210 & 28.3879 \\
\hline $\mathbf{1 0}$ & 2.1083 & 26.7969 & 27.3205 & 1.4519 & 29.0827 & 28.5163 \\
\hline $\mathbf{1 5}$ & 2.1276 & 26.8037 & 27.3167 & 1.4586 & 29.2328 & 28.5870 \\
\hline $\mathbf{2 0}$ & 2.1437 & 26.8140 & 27.3337 & 1.4605 & 29.3946 & 28.5056 \\
\hline $\mathbf{2 5}$ & 2.1491 & 26.8288 & 27.3507 & 1.4627 & 29.4482 & 28.3900 \\
\hline $\mathbf{3 0}$ & 2.1551 & 26.8516 & 27.3866 & 1.4681 & 29.5804 & 28.4018 \\
\hline $\mathbf{3 5}$ & 2.1672 & 26.8766 & 27.4131 & 1.4706 & 29.6817 & 28.4511 \\
\hline $\mathbf{4 0}$ & 2.1684 & 26.8937 & 27.4490 & 1.4718 & 29.8083 & 28.5158 \\
\hline $\mathbf{4 5}$ & 2.1804 & 26.9245 & 27.4905 & 1.4766 & 29.8984 & 28.5901 \\
\hline $\mathbf{5 0}$ & 2.1944 & 26.9416 & 27.5189 & 1.4813 & 29.9546 & 28.6534 \\
\hline
\end{tabular}


The surface behavior of any material can be investigated by measuring its reflectivity. The reflectivity versus energy for NCF at $(0,5,10,15,20,25,30,35,40,45$ and 50) GPa is
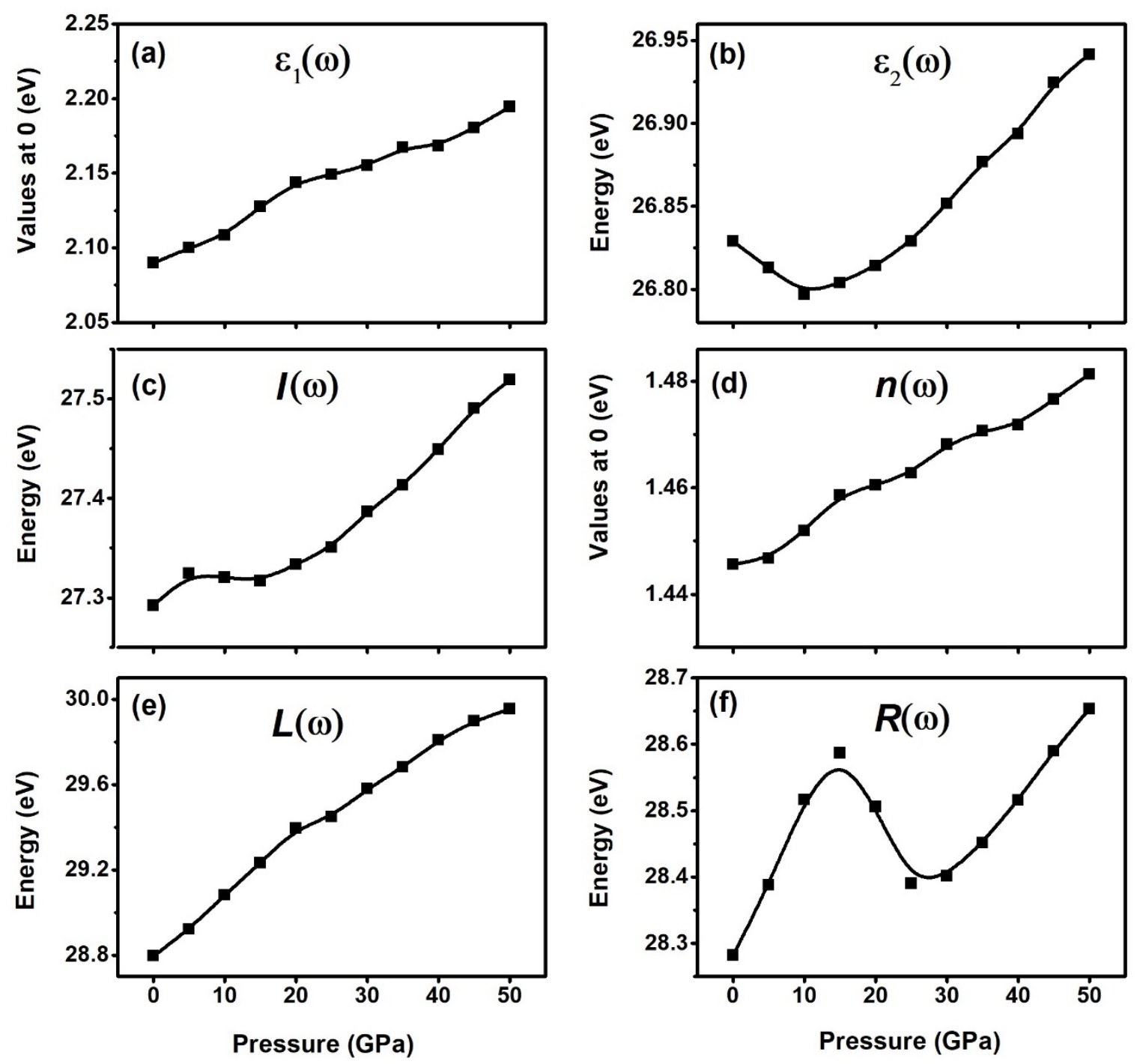

Fig. 9. Trend of different optical properties. (a) $\varepsilon_{1}(\omega)$, (b) $\varepsilon_{2}(\omega)$, (c) $I(\omega)$, (d) $n(\omega)$, (e) $L(\omega)$ and (f) $R(\omega)$ versus external isotropic pressure. The values of $\varepsilon_{1}(\omega)$ and $n(\omega)$ are taken at $0 \mathrm{eV}$.

shown in Fig. 8f. It reveals that at zero frequency, reflectivity for NCF is equal to 0.0332 , $0.0330,0.0340,0.0348,0.0357,0.0344,0.0351,0.0357,0.0364,0.0370$ and 0.0376 at $(0$, $5,10,15,20,25,30,35,40,45$ and 50) GPa, respectively. Then it starts increasing from these zero limits with increase in pressure where the highest peak of reflectivity is achieved at $28.2819 \mathrm{eV}, 28.3879 \mathrm{eV}, 28.5163 \mathrm{eV}, 28.5870 \mathrm{eV}, 28.5056 \mathrm{eV}, 28.3900 \mathrm{eV}$, 
$28.4018 \mathrm{eV}, 28.4511 \mathrm{eV}, 28.5158 \mathrm{eV}, 28.5901 \mathrm{eV}$ and $28.6534 \mathrm{eV}$ for $(0,5,10,15,20,25$, $30,35,40,45$ and 50) GPa, respectively.

The energy loss function $L(\omega)$ is an imperative factor which gives information about how much energy has been lost for the electron which is moving inside the material is shown in Fig.8e for NCF. The energy loss function $L(\omega)$ has peaks that precisely describe features associated with plasma resonance and therefore, the connected frequency is acknowledged as plasma frequency. At zero pressure the value of highest peak is $28.7962 \mathrm{eV}$ and exceeds this value as pressure increases from $5 \mathrm{GPa}-50 \mathrm{GPa}$. The characteristics associated to plasma resonance can be described through peaks in energy loss function $L(\omega)$ and thus the associated frequency is named as plasma frequency.

For NCF system, both without and with pressure, the value of static-refractive index $n(\omega)$ for zero photon energy, has been calculated and seems merely the same which is 1.4456 (Fig. 8d). Before and after applying pressure, there is a slight shifting of $n(\omega)$ which favors the semiconducting behavior of ternary compound (Fig. 9d). When photon-energy is zero, the lowest absorption energy value corresponds to $n(\omega)$. At the energy point where absorption peak is maximum the value of refractive index is lowest. A material will be efficiently applicable in photovoltaic system whose optical conductivity; absorption and refractive index are high. It is also used in optical devices including waveguides, data storage media, photonic crystals and so on [31].

\section{Conclusion}

Current paper aimed a comprehensive theoretical investigation of the outcomes of externally applied pressure from $0 \mathrm{GPa}-50 \mathrm{GPa}$ with 5 step increment i.e. $(0,5,10,15,20$, $25,30,35,40,45$ and 50) $\mathrm{GPa}$ on optical, electronic and structural properties of $\mathrm{NaCaF}_{3}$ by using density functional theory (DFT) based CASTEP code, through ultra-soft pseudopotential USP plane wave and Perdew Burke Ernzerhof (PBE) exchange correlation functional of Generalized-Gradient-Approximation (GGA), has been explored. The structural parameters and unit cell volume are computed after geometry optimization, which are comparable to already reported results. The valence band is majorly controlled by $p$-states of $\mathrm{F}$ and $\mathrm{Na}$. The electronic bandgap exhibits an increasing trend $4.773 \mathrm{eV}$ - 
$6.203 \mathrm{eV}$ with increasing external pressure. The sharp increase in bandgap is noticed up to $20 \mathrm{GPa}$ as compared to higher external pressures. Moreover, the nature of the band gap for all values of external pressures remained same i. e. direct bandgap. The mystery of increasing band gap is nicely decoded by total-density of states (TDOS) and elemental partial-density of states (EPDOS). We have also calculated different optical properties like the energy-loss function, refractive index, the reflectivity as well as absorption function for different pressures to analyze the result in optical properties by increasing bandgap. It is seen that energy loss function highest peak acknowledged as Plasmon peak and shifts towards higher energies $(28.7962 \mathrm{eV}-29.9546 \mathrm{eV})$ i. e. blue shift for different pressures which confirms the increment of bandgap. At zero photon energy for all applied pressures the static refractive index $n(\omega)$ has nearly the same value i. e. $1.4456 \mathrm{eV}$. Before and after applying pressure, there is a slight shifting of $n(\omega)$ which favors the semiconductor behavior of ternary compound. A material will be efficiently applicable in photovoltaic system whose optical conductivity; absorption and refractive index are high. It is also used in optical devices including waveguides, data storage media, and photonic crystals and so on.

\section{Declaration of Competing Interest}

The authors declare that they have no known competing financial interests or personal relationships that could have appeared to influence the work reported in this paper.

\section{Acknowledgment}

S. S. A. Gillani and Riaz Ahmad would like to thank Higher Education Commission (HEC) of Pakistan for financial support under grant numbers 6934/HEC/NRPU, 6958/HEC/NRPU, respectively.

\section{References}

[1] H.-R. Wenk and A. Bulakh, Minerals: Their Constitution and Origin (Cambridge University Press, New York, 2004).

[2] Soleimanpour, S., and F. Kanjouri, Elastic, electronic and optical properties of the cubic fluoro-perovskite $\mathrm{KCaF}_{3}$ under pressure. Indian Journal of Physics, 89(7) (2015), pp. 687697. 
[3] T Nishimatsu et al, Band Structures of Perovskite-Like Fluorides for VacuumUltraviolet-Transparent Lens Materials, Jpn. J. Appl. Phys. 41 (2002), pp. 365.

[4] Banwari Lal, S. K. Khosa, Dielectric characteristics of melt grown doped $\mathrm{KMgF}_{3}$ crystals, Materials Chemistry and Physics 83 (2004), pp. 158-168.

[5] G. H. Jonker and J. H. Van Santen, Ferromagnetic Compounds of Manganese with perovskite structure, Physica 16 (1950), pp. 337-349.

[6] M. Eibschtitz, H. J. Guggenheim, Antiferromagnetic-Piezoelectric crystals: $\mathrm{BaMe}_{4}$ (M $=\mathrm{Mn}, \mathrm{Fe}, \mathrm{Co}$, and Ni), Solid State Commun. 6 (1968), pp. 737-739.

[7] A. H. Cooke, D. A. Jones, and Ferromagnetism in lithium holmium fluoride-LiHoF4: I. Magnetic measurements, Journal of Phys. C: Solid State Phys. 8(1975), pp. 4083-4088.

[8] Mousa, A. A, First-principles study of structural, electronic, and optical properties of the $\mathrm{KCaX}_{3}(\mathrm{X}=\mathrm{F}$ and $\mathrm{Cl})$ compounds, International Journal of Modern Physics B, 28(21) (2014), p. 1450139.

[9] S. Soleimanpour, F. Kanjouri, Elastic, electronic, and optical properties of the cubic fluoro-perovskite $\mathrm{KCaF}_{3}$ under pressure, Indian Journal of Physics, 89(7), (2015), pp. 687697.

[10] I. Zeba, R. Jabeen, Riaz Ahmad, M. Shakil, M. Rafique, M. Rizwan, N. Bashir, S. S. A. Gillani, Effect of anomalous behavior of Be-doping on structural stability, bandgap and optical properties in comparison with $\mathrm{Mg}$-doped $\mathrm{BaZrO}_{3}$ perovskite: insights from DFT calculations, Optical and Quantum Electronics, 54 (2020), pp. 234.

[11] X. Yang, Q. J. Li, R. Liu, B. Liu, H. F. Zhang, S.Q. Jiang, J. Liu, B. Zou, T. Cui, B. B. Liu, Structural phase transition of $\mathrm{BaZrO}_{3}$ under high pressure, J. Appl. Phys. 115 (2014), pp. 124907.

[12] Yang, X., Wang, Y., Song, Q., Chen, Y., \& Xue, Y. H, Pressure Effects on structural, electronic, elastic and optical properties of cubic and tetragonal phases of $\mathrm{BaZrO}_{3}$, Acta Physica Polonica, A., 133(5) (2018).

[13] Jin, Z., Wu, Y., Li, S., Chen, S., Zhang, W., Wu, Q., \& Zhang, C. (2020). Firstprinciples calculation of the electronic structure, optical, elastic, and thermodynamic properties of cubic perovskite $\mathrm{LiBeF}_{3}$. Materials Research Express, 6(12), pp. 125116.

[14] M. W. Shafer, The synthesis and characterization of vanadium difluoride, $\mathrm{NaVF}_{3}$, $\mathrm{KVF}_{3}$, and $\mathrm{RbVF}_{3}$, Material Research Bulletin 4 (1969), pp. 905-912. 
[15] D.K. Sardar, W.A. Sibley, R. Aicala, Optical absorption and emission from irradiated $\mathrm{RbMgF}_{3}: \mathrm{Eu}_{2}{ }^{+}$and $\mathrm{KMgF}_{3}: \mathrm{Eu}_{2}{ }^{+}$, J. Lumin. 27 (1982), pp. 401-411.

[16] Georg K.H. Madsen, Peter Blaha, Efficient linearization of the augmented plane-wave method, Phys. Rev. B 64 (2001), pp. 1951341-1951349.

[17] J.P. Perdew, K. Burke, M. Ernzerhof, generalized gradient approximation made simple, Phys. Rev. Lett. 77 (1996), pp. 3865.

[18] J. P. Perdew, K. Burke, M. Ernzerhof, generalized gradient approximation made simple, Phys. Rev. Lett. 77 (1996), pp. 3865.

[19] J. P. Perdew, J. A. Chevary, S. H. Vosko, K.A. Jackson, M. R. Pederson, D. J. Singh, C. Fiolhais, Atoms, molecules, solids, and surfaces: applications of the generalized gradient approximation for exchange and correlation, Phys. Rev. B 46 (1992), pp. 6671.

[20] M. D. Segall, P. L. D. Lindan, M. J. Probert, C. J. Pickard, P. J. Hasnip, S. J. Clark, M. C. Payne, First principles simulation: ideas, illustrations and the CASTEP code, J. Phys. Condens. Matter 14 (2002), pp. 2717.

[21] P. Hohenberg, W. Kohn, Inhomogeneous electron gas, Phys. Rev. 136 (1964), pp. 864.

[22] W. Kohn, L. J. Sham, Self-consistent equations including exchange and correlation effects. Phys. Rev. A 140 (1965), pp. 1133.

[23] W. Kohn, Nobel lectures: electronic structure of matter-wave functions and density functionals, Rev. Mod. Phys. 71 (1999), pp. 1253.

[24] Hendrik J. Monkhorst, James D. Pack, Special points for Brillion-zone integrations, Physical Review B 13 (1976), pp. 5188-5192.

[25] Thomas H. Fischer, General Methods for Geometry and Wave Function Optimization, J. Phys. Chem. 96 (1992), pp. 9768-9774.

[26] H. Bouafia, B. Sahli, S. Hiadsi, B. Abidri, D. Rached, A. Akriche, M. N. Mesli, Theoretical investigation of structural, elastic, electronic, and thermal properties of $\mathrm{KCaF}_{3}$, $\mathrm{K}_{0.5} \mathrm{Na}_{0.5} \mathrm{CaF}_{3}$ and $\mathrm{NaCaF}_{3}$ perovskites, Superlatt. Microstruct. 82 (2015), pp. 525-537.

[27] M. J. Mehl, Pressure dependence of the elastic moduli in aluminum-rich Al-Li compounds, Phys. Rev. B 47 (1993), pp. 2493-2500.

[28] M. Harmel, H. Khachai, M. Ameri, R. Khenata, N. Baki, A. Haddou, B. Abbar, S, Ugur, S. Bin Omran, F. Soyalp, DFT-based ab initio study of the electronic and optical 
properties of cesium based fluoro-perovskite $\mathrm{CsMF}_{3}(\mathrm{M}=\mathrm{Ca}$ and $\mathrm{Sr}$ ), Modern Phys. B 26 (2012), pp. 12501991-125019913.

[29] M. H. Benkabou, M. Harmel, A. Haddou, A. Yakoubi, N. Baki, R. Ahmed, Y. AlDouri, S.V. Syrotyuk, H. Khachai, R. Khenata, C. H. Voon, M. R. Johan, Structural, electronic, optical and thermodynamic investigations of $\mathrm{NaXF}_{3}(\mathrm{X}=\mathrm{Ca}$ and $\mathrm{Sr})$ : Firstprinciples calculations, Chinese Journal of Physics 56 (2018), pp. 131-144.

[30] A. I. Lebedev, $\mathrm{Ab}$ initio Calculations of Phonon Spectra in $\mathrm{ATiO}_{3}$ Perovskite Crystals (A = Ca, Sr, Ba, Ra, Cd, Zn, Mg, Ge, Sn, Pb), Physics of the Solid State, 51 (2009), pp. 362-372.

[31] Jin Yang, Lijan Yang, Theoretical investigation of electronic structure, optical, elastic, hardness and thermodynamics properties of jadeite, Material science in semiconductor processing, 31 (2015), pp. 509-516. 
Figures

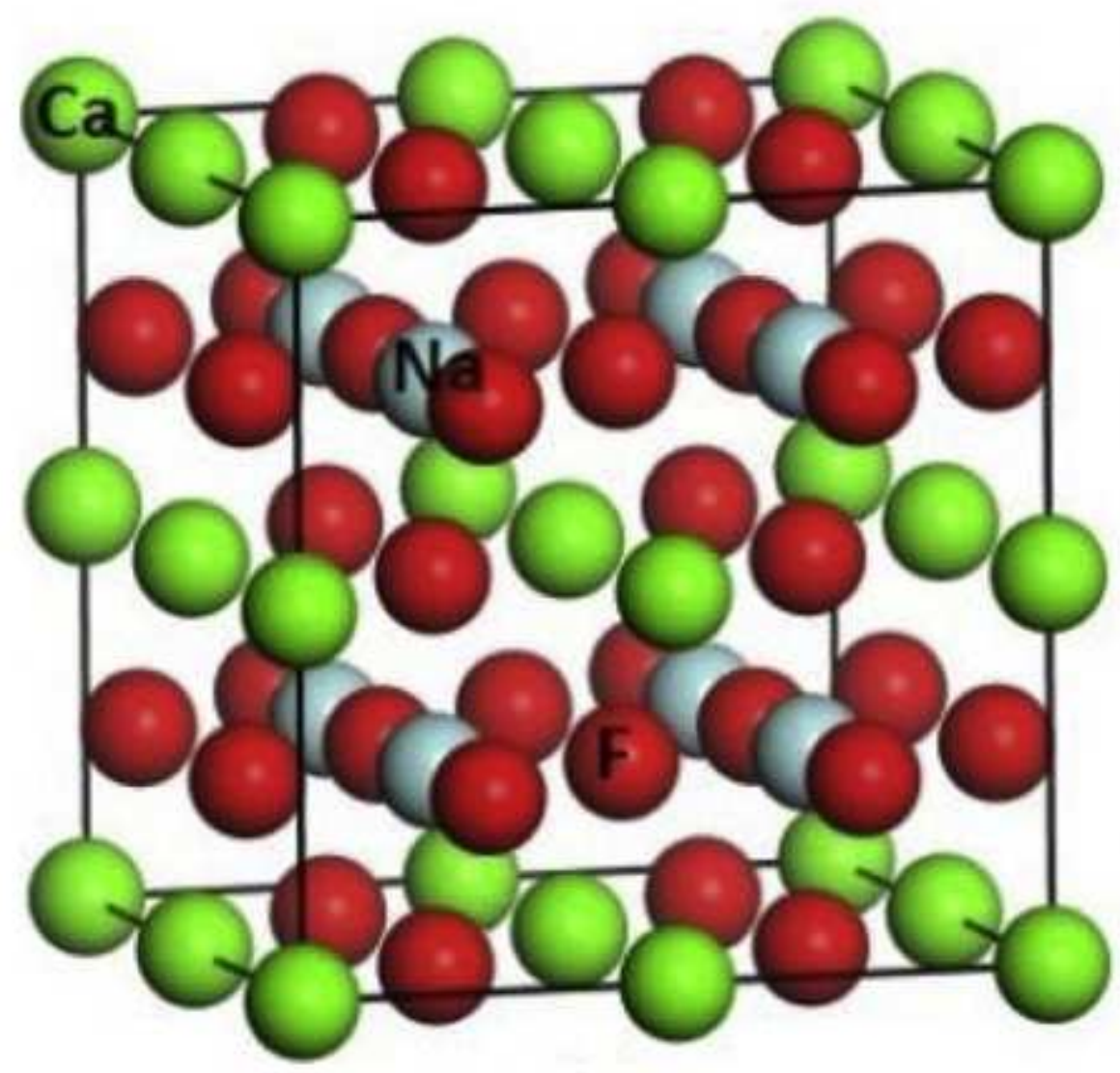

Figure 1

$2 \times 2 \times 2$ supercell of NCF. 


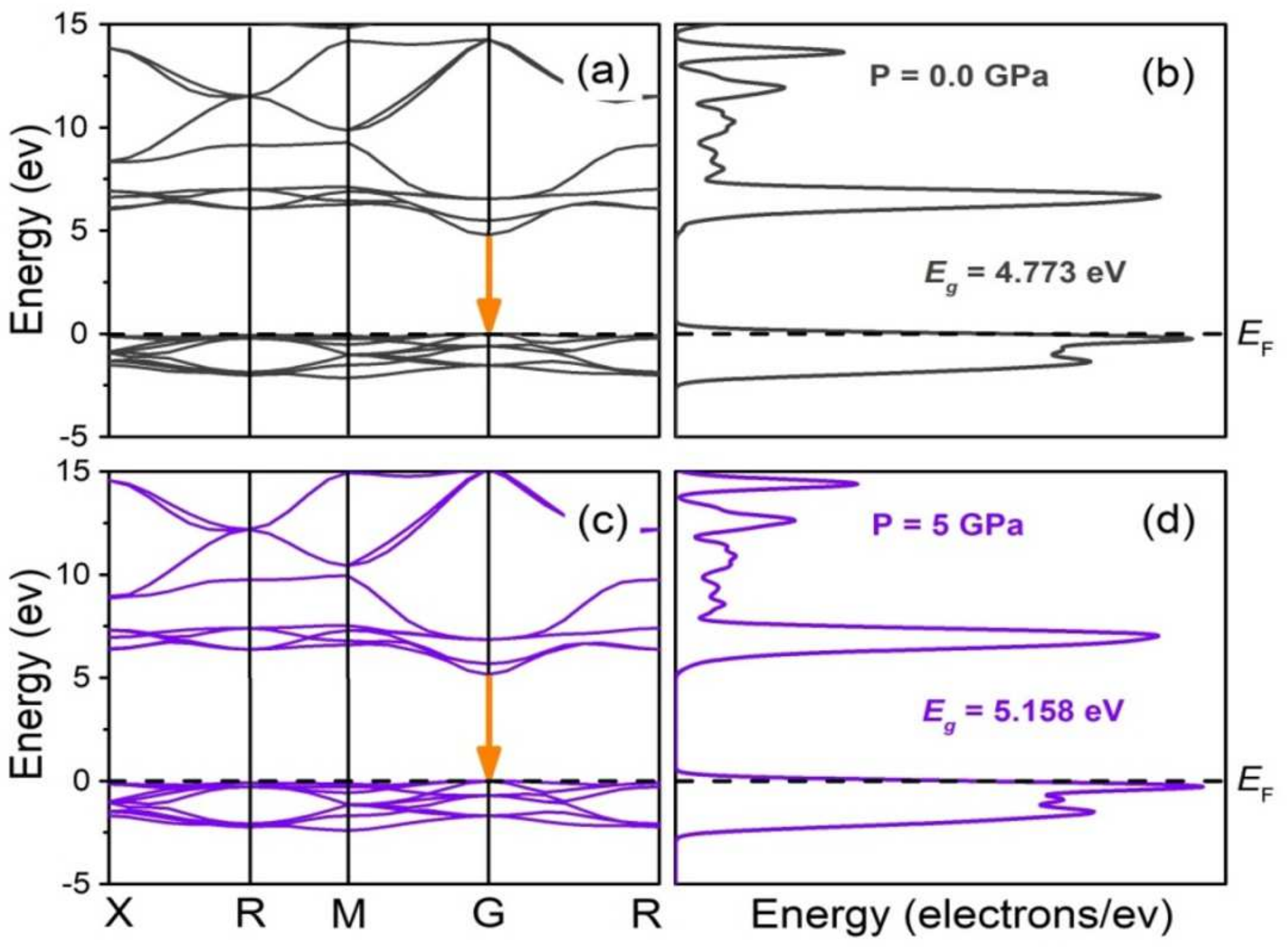

Figure 2

At OGPa, (a) energy band structure and (b) DOS of NCF. At 5 GPa, (c) energy band structure and (d) DOS of NCF. 

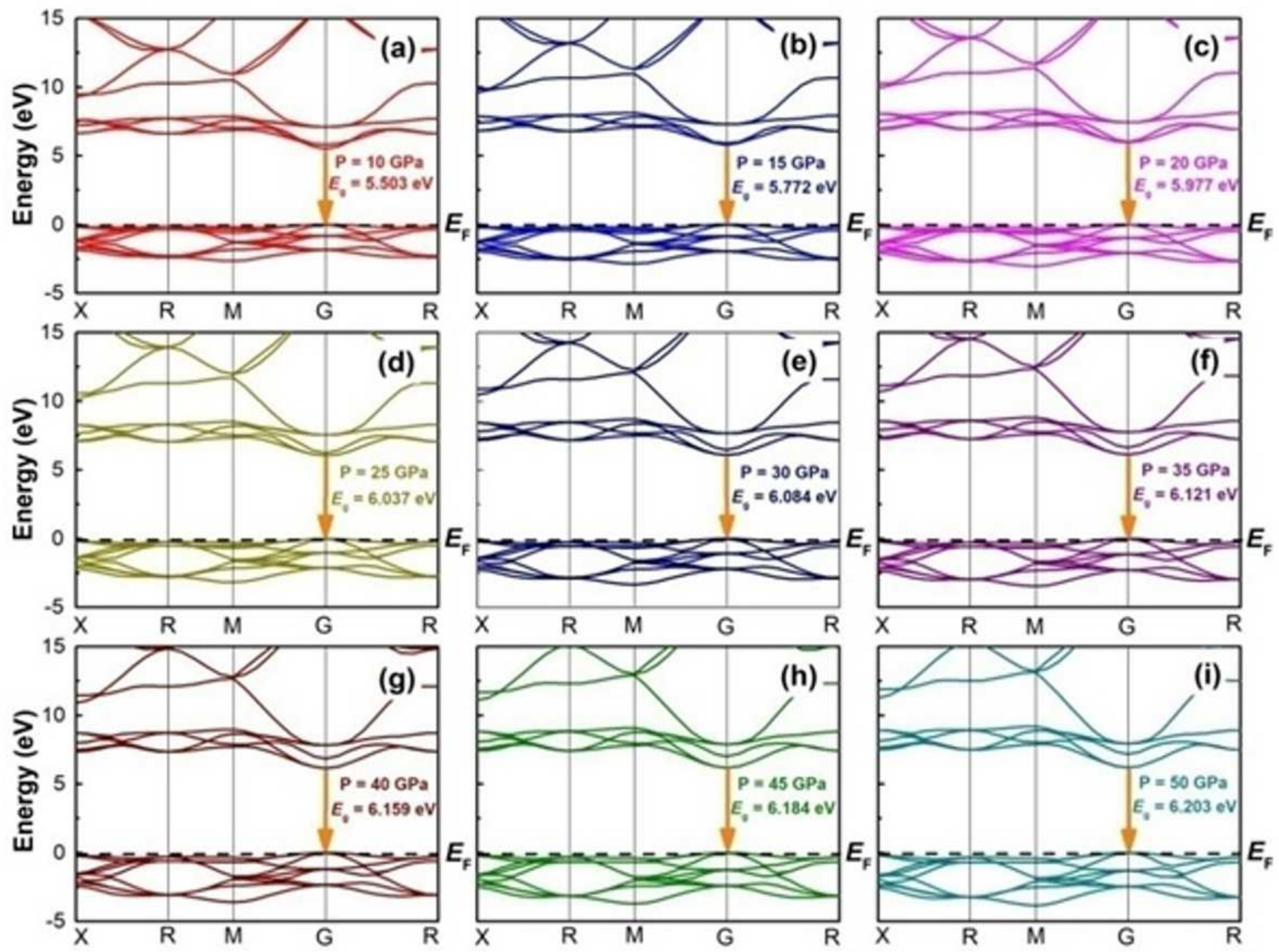

Figure 3

(a-i) The energy band spectrum at different pressure (10 GPa - $50 \mathrm{GPa}$ ), respectively. 


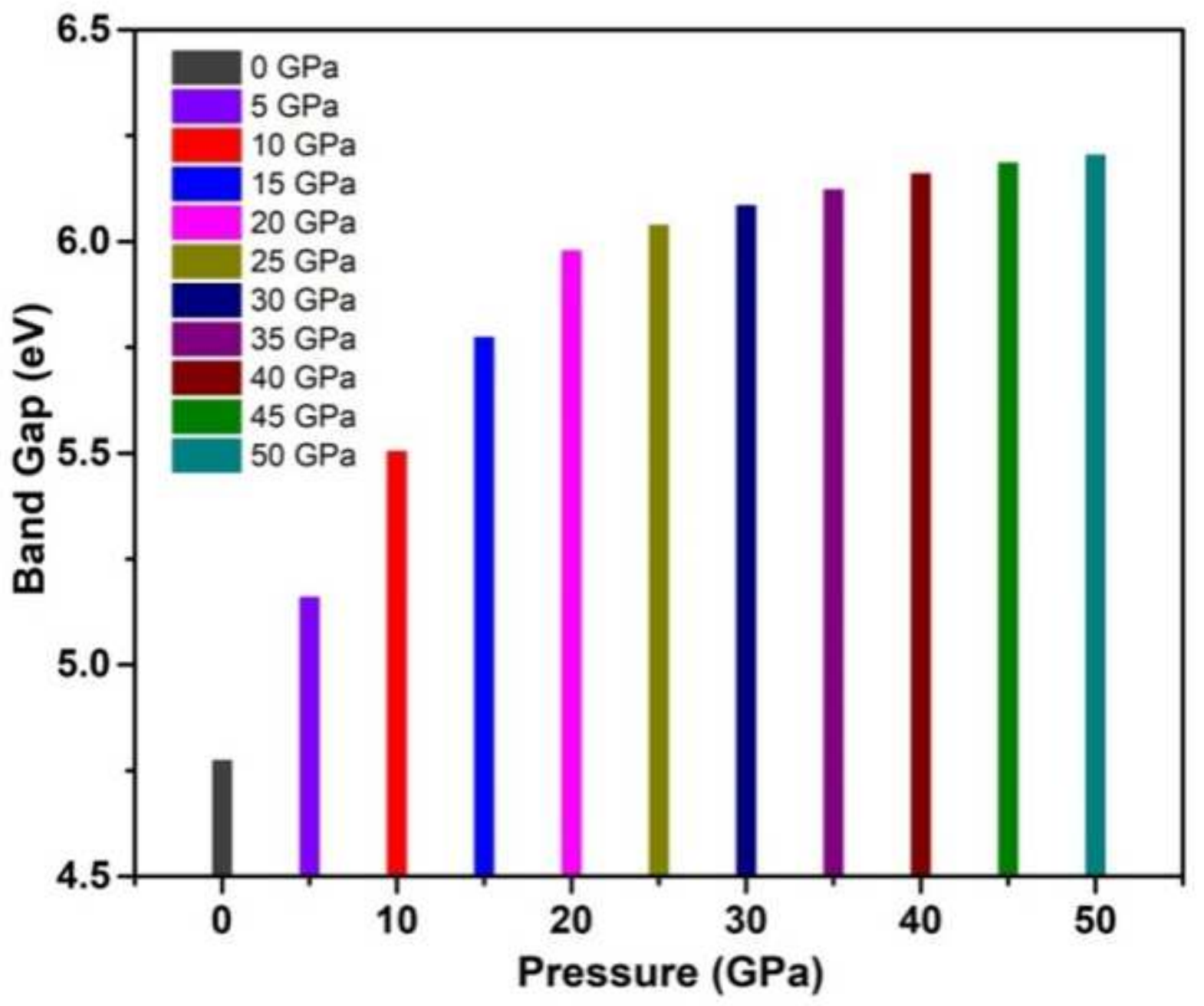

Figure 4

Value of bandgaps of $\mathrm{NaCaF} 3$ with pressure which is showing increasing trend. 

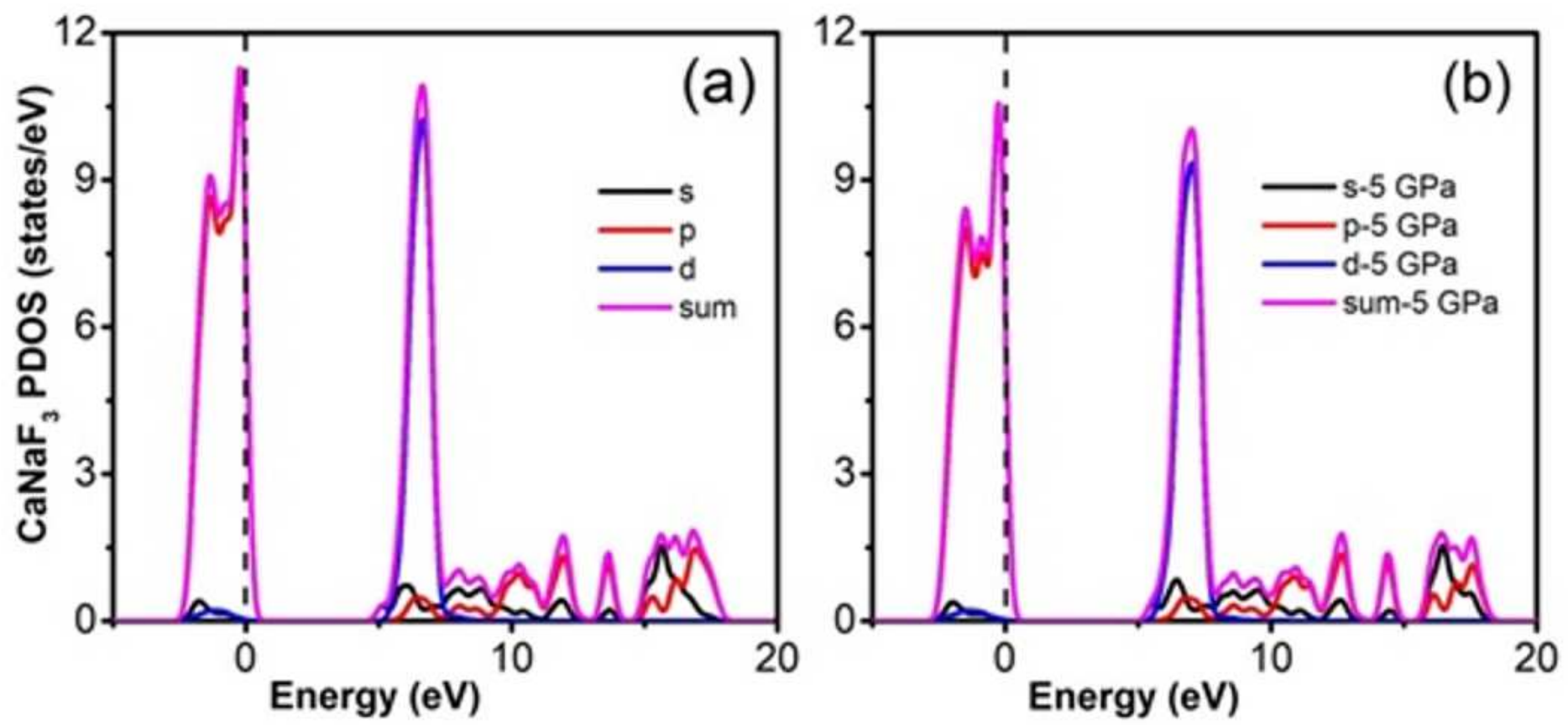

Figure 5

Partial density of states (PDOS) of NaCaF3 at pressure (a) $0 \mathrm{GPa}$ and (b) $5 \mathrm{GPa}$. 

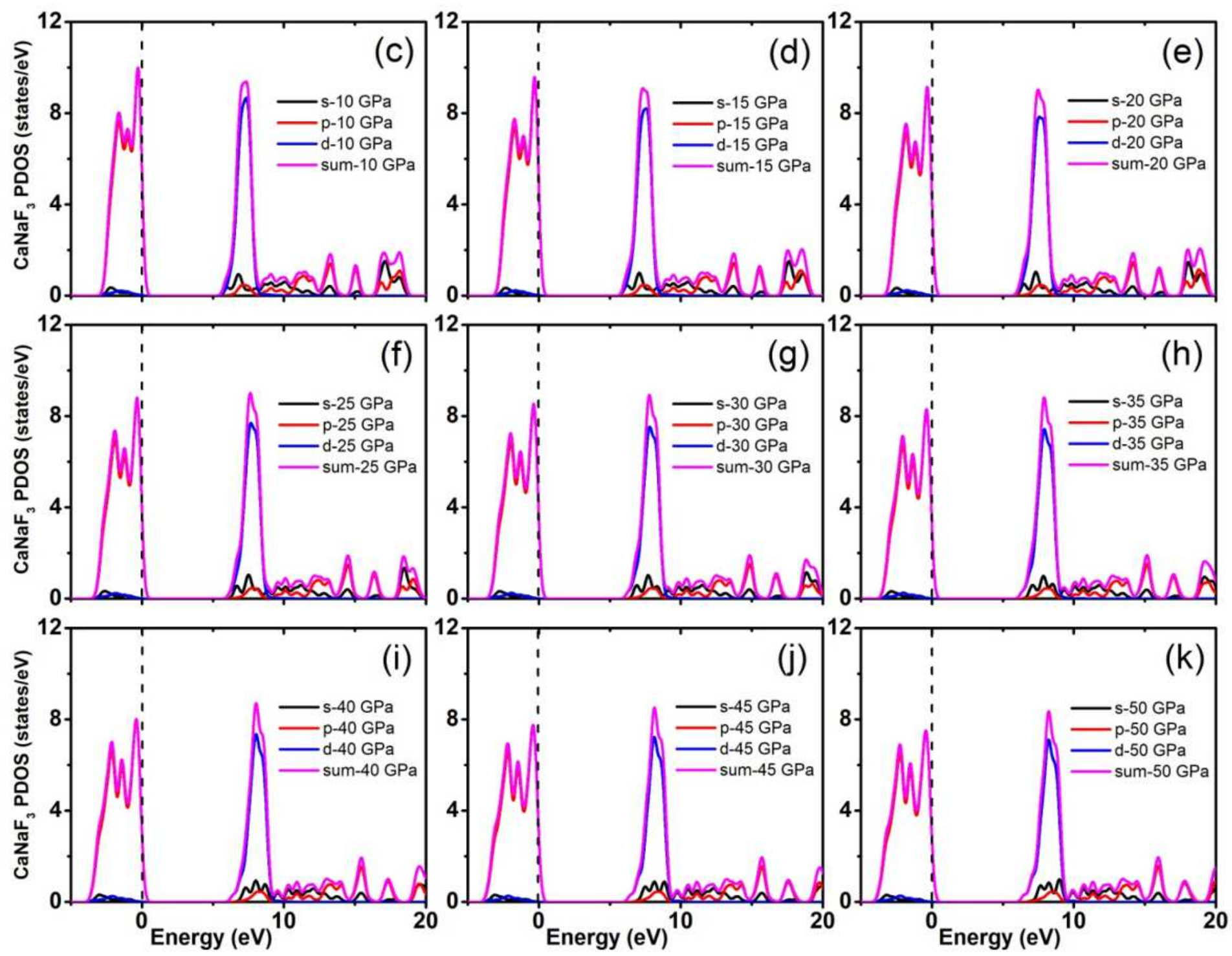

Figure 6

Partial density of states of $\mathrm{NaCaF} 3$ under different pressure ranging $10 \mathrm{GPa}-50 \mathrm{GPa}$.

Image not available with this version

Figure 7

Figure not provided in this version 

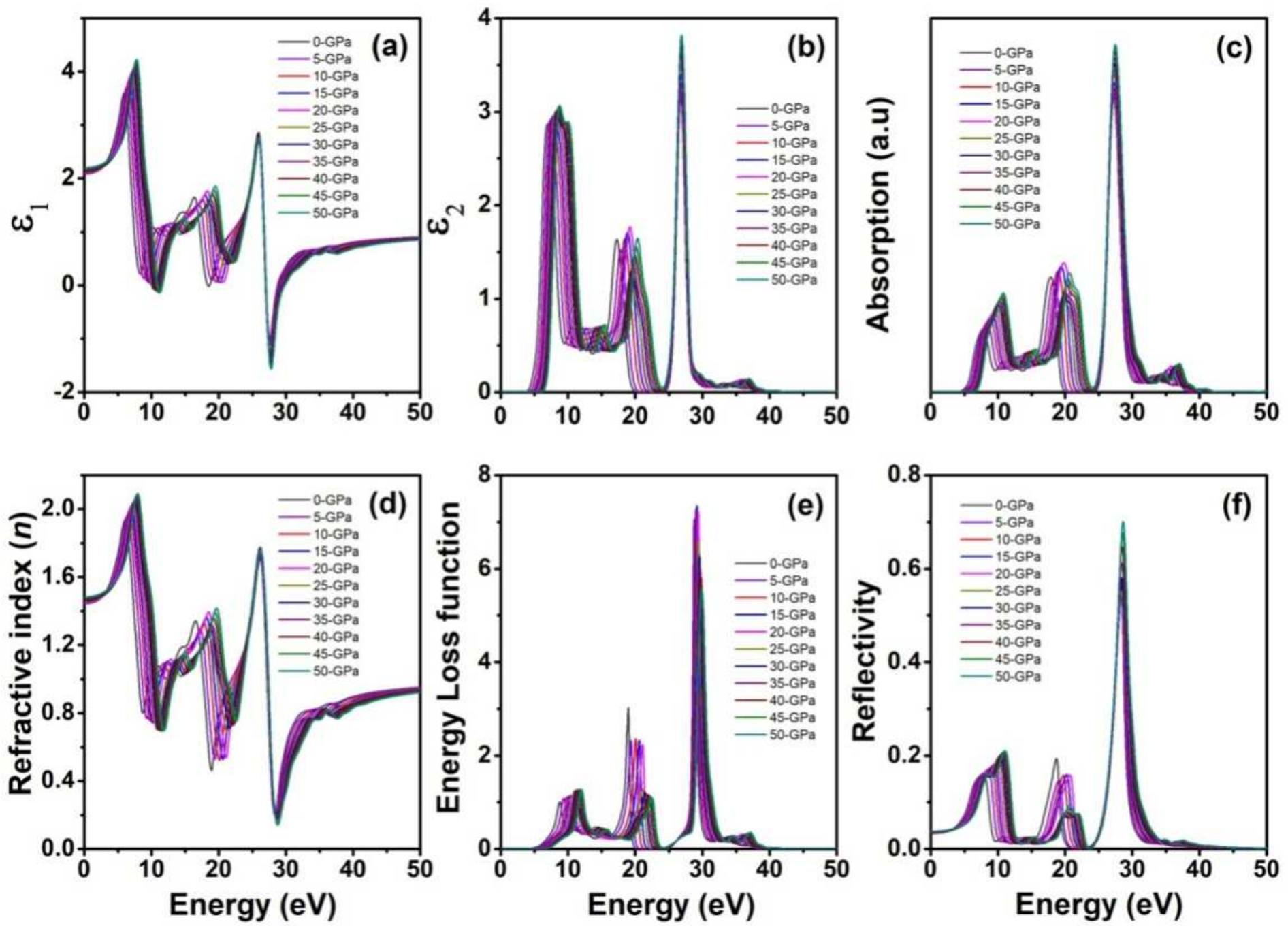

Figure 8

Optical properties of NaCaF3 with inclusion and exclusion of a variety of pressure. $(a, b)$ Dielectric function consist of real and imaginary part (c) absorption spectra, (d) refractive index, (e) energy-loss function and (f) reflectivity. 

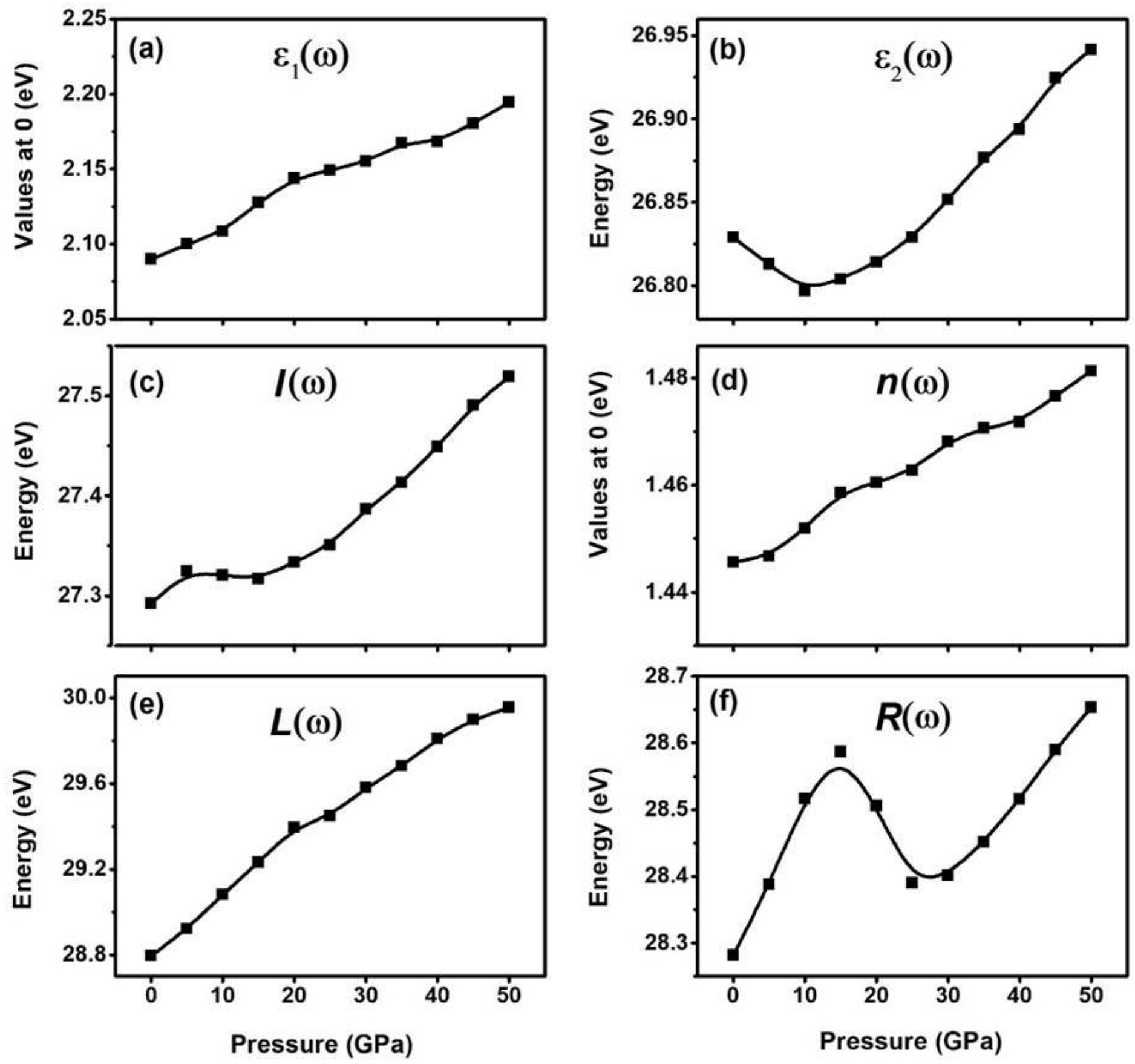

Figure 9

Trend of different optical properties. (a) $\varepsilon_{-} 1(\omega),(b) \varepsilon_{-} 2$ ( $(\omega)$, (c) $I(\omega),(d) n(\omega),(e) L(\omega)$ and (f) $R(\omega)$ versus external isotropic pressure. The values of $\varepsilon_{-} 1(\omega)$ and $n(\omega)$ are taken at $0 \mathrm{eV}$. 